PROCEEDINGS OF THE

AMERICAN MATHEMATICAL SOCIETY

Volume 129, Number 4, Pages 1015-1020

S 0002-9939(00)05793-2

Article electronically published on October 11, 2000

\title{
ESSENTIALITY FOR MÖNCH TYPE MAPS
}

\author{
RAVI P. AGARWAL AND DONAL O'REGAN \\ (Communicated by Jonathan M. Borwein)
}

\begin{abstract}
A new fixed point theorem for Mönch maps on locally convex spaces is given. In addition, a continuation theorem for Mönch maps is presented.
\end{abstract}

\section{INTRODUCTION}

In 1980 Mönch presented a fixed point theorem, for maps between Banach spaces, which extends the Schauder and, more generally, Sadovskii fixed point theorems. The fixed point theorem of Mönch has been particularly useful in establishing existence of solutions to nonlinear boundary value problems in Banach spaces (see [5, 7)). Our paper has two main goals. The first is to extend Mönch's theorem to the locally convex space setting, and the second is to show that the property of having a fixed point is invariant by homotopy for Mönch type maps. More precisely we show that if the zero map is essential and $F \cong 0$, then $F$ is essential. Our results improve, extend and complement previously known results [1, 4, 7, 9].

\section{MÖNCH TYPE MAPS}

We begin by presenting a continuation theory for Mönch type maps. Let $E$ be a Hausdorff linear topological space, $U$ an open subset of $E$ and $0 \in U$.

Definition 2.1. We let $M(\bar{U}, E)$ denote the set of all continuous maps $F: \bar{U} \rightarrow$ $E$ which satisfy Mönch's condition (i.e. if $C \subseteq \overline{c o}(\{0\} \cup F(C))$ and $C \subseteq \bar{U}$ is countable, then $\bar{C}$ is compact); here $\bar{U}$ denotes the closure of $U$ in $E$.

Definition 2.2. We let $F \in M_{\partial U}(\bar{U}, E)$ if $F \in M(\bar{U}, E)$ with $x \neq F(x)$ for $x \in \partial U$; here $\partial U$ denotes the boundary of $U$ in $E$.

Definition 2.3. A map $F \in M_{\partial U}(\bar{U}, E)$ is essential if for every $G \in M_{\partial U}(\bar{U}, E)$ with $\left.G\right|_{\partial U}=\left.F\right|_{\partial U}$ there exists $x \in U$ with $x=G(x)$.

Theorem 2.1. Let $E$ be a Hausdorff linear topological space, $U$ an open subset of $E$ and $0 \in U$. Suppose $F \in M(\bar{U}, E)$ with

$$
\text { the zero map is essential in } M_{\partial U}(\bar{U}, E)
$$

and

$$
x \neq \lambda F(x) \text { for any } x \in \partial U \text { and } \lambda \in(0,1]
$$

Received by the editors June 15, 1999.

2000 Mathematics Subject Classification. Primary 47H10. 
holding. Also assume that one of the following conditions holds:

$$
E \text { is a normal space }
$$

or

$$
\left\{\begin{array}{l}
E \text { is such that any closed subset is compact } \\
\text { iff it is sequentially compact. }
\end{array}\right.
$$

Then $F$ is essential in $M_{\partial U}(\bar{U}, E)$.

Proof. Let $H \in M_{\partial U}(\bar{U}, E)$ with $\left.H\right|_{\partial U}=\left.F\right|_{\partial U}$. We must show $H$ has a fixed point in $U$. Consider

$$
B=\{x \in \bar{U}: x=t H(x) \text { for some } t \in[0,1]\} .
$$

Now $B \neq \emptyset$ since $0 \in U$ and $B$ is closed since $H$ is continuous. In addition, $B \cap \partial U=\emptyset$ since $(2.2)$ holds and $\left.H\right|_{\partial U}=\left.F\right|_{\partial U}$. We now claim that there exists a continuous $\mu: \bar{U} \rightarrow[0,1]$ with $\mu(\partial U)=0$ and $\mu(B)=1$. The claim is immediate if (2.3a) holds since $B$ and $\partial U$ are closed. Next suppose (2.3b) holds. Since Hausdorff linear topological spaces are completely regular [6], the claim will be true if we show $B$ is compact. To see this let $\left\{x_{n}\right\}_{1}^{\infty}$ be any sequence in $B$. Then there exists a sequence $\left\{t_{n}\right\}_{1}^{\infty}$ of $[0,1]$ with $x_{n}=t_{n} H\left(x_{n}\right)$ for $n \in\{1,2, \cdots\}$. Without loss of generality assume $t_{n} \rightarrow t \in[0,1]$. Let $C=\left\{x_{n}\right\}_{n=1}^{\infty}$. Notice $C$ is countable and since $x_{n}=t_{n} H\left(x_{n}\right)+\left(1-t_{n}\right) 0$ for $n \in\{1,2, \cdots\}$ we have

$$
C \subseteq \overline{c O}(\{0\} \cup H(C)) .
$$

Since $H \in M(\bar{U}, E)$, we have that $\bar{C}$ is compact. From (2.3b) there exists a subsequence $N$ of $\{1,2, \cdots\}$ and an $x \in \bar{C}$ with

$$
x_{n} \rightarrow x \text { as } n \rightarrow \infty \text { in } N \text {. }
$$

This together with $x_{n}=t_{n} H\left(x_{n}\right)$ for $n \in N$, and the continuity of $H$, yields $x=t H(x)$ and so $x \in B=\bar{B}$. Consequently $B(=\bar{B})$ is sequentially compact, so is compact from $(2.3 \mathrm{~b})$.

Define a map $R_{\mu}: \bar{U} \rightarrow E$ by

$$
R_{\mu}(x)=\mu(x) H(x) .
$$

We first show $R_{\mu}$ satisfies the Mönch condition. Let $C \subseteq \bar{U}$ be countable and

$$
C \subseteq \overline{c o}\left(\{0\} \cup R_{\mu}(C)\right) .
$$

Now since $R_{\mu}(x)=\mu(x) H(x)$ we have $R_{\mu}(C) \subseteq c o(\{0\} \cup H(C))$. In addition, since $c o(\{0\} \cup H(C))$ is convex and $\{0\} \cup c o(\{0\} \cup H(C))=c o(\{0\} \cup H(C))$ we have that

$$
C \subseteq \overline{c o}\left(\{0\} \cup R_{\mu}(C)\right) \subseteq \overline{c o}(c o(\{0\} \cup H(C)))=\overline{c o}(\{0\} \cup H(C)) .
$$

Since $H \in M(\bar{U}, E)$ we have that $\bar{C}$ is compact. Thus $R_{\mu} \in M(\bar{U}, E)$ with $\left.R_{\mu}\right|_{\partial U}=0$. Now since the zero map is essential in $M_{\partial U}(\bar{U}, E)$ there exists $x \in U$ with $x=R_{\mu}(x)$. Consequently, $x \in B$ and so $\mu(x)=1$, i.e. $x=H(x)$.

Before we give an example of a zero essential map in $M_{\partial U}(\bar{U}, E)$ we present another result which is useful in applications. Our result extends [1, Theorem 2.1]. 
Theorem 2.2. Let $E$ be a Hausdorff linear topological space, $U$ an open subset of $E$ and $0 \in U$. Suppose $F \in M_{\partial U}(\bar{U}, E)$ is an essential map and $H: \bar{U} \times[0,1] \rightarrow E$ is a continuous map with the following properties:

$$
\begin{gathered}
H(x, 0)=F(x) \text { for } x \in \bar{U} \\
\left\{\begin{array}{l}
x \neq H_{t}(x) \text { for any } x \in \partial U \text { and } t \in(0,1] \\
\left(\text { here } H_{t}(x)=H(t, x)\right)
\end{array}\right.
\end{gathered}
$$

and

$$
\left\{\begin{array}{l}
\text { if } C \subseteq \bar{U} \text { is countable and } C \subseteq \overline{c o}(\{0\} \cup H(C \times[0,1])) \\
\text { then } \bar{C} \text { is compact. }
\end{array}\right.
$$

Also assume either (2.3a) or (2.3b) occurs. Then $H_{1}$ has a fixed point in $U$.

Proof. Let

$$
B=\left\{x \in \bar{U}: x=H_{t}(x) \text { for some } t \in[0,1]\right\} .
$$

When $t=0, H_{0}=F$ and since $F \in M_{\partial U}(\bar{U}, E)$ is essential, there exists $x \in U$ with $x=F(x)$. Thus $B \neq \emptyset$. Also $B$ is closed and $B \cap \partial U=\emptyset$. As in Theorem 2.1 (use (2.6)) there exists a continuous $\mu: \bar{U} \rightarrow[0,1]$ with $\mu(\partial U)=0$ and $\mu(B)=1$. Define a map $R_{\mu}: \bar{U} \rightarrow E$ by

$$
R_{\mu}(x)=H(x, \mu(x)) .
$$

Now $R_{\mu}$ is continuous and satisfies Mönch's condition (use (2.6)). Moreover for $x \in \partial U$ we have $R_{\mu}(x)=H_{0}(x)=F(x)$. Thus $R_{\mu} \in M_{\partial U}(\bar{U}, E)$ with $\left.R_{\mu}\right|_{\partial U}=$ $\left.F\right|_{\partial U}$. Since $F \in M_{\partial U}(\bar{U}, E)$ is essential there exists $x \in U$ with $x=R_{\mu}(x)$ (i.e. $\left.H_{\mu(x)}(x)=x\right)$. Thus $x \in B$ and so $\mu(x)=1$, i.e. $x=H_{1}(x)$.

Remark 2.1. If (2.3a) occurs it is possible to replace (2.6) with the following condition:

$$
\left\{\begin{array}{l}
\text { for any continuous } \mu: \bar{U} \rightarrow[0,1] \text { with } \mu(\partial U)=0 \text { the map } \\
R_{\mu}: \bar{U} \rightarrow E \text { defined by } R_{\mu}(x)=H(x, \mu(x)) \text { satisfies Mönch's } \\
\text { condition (i.e. if } C \subseteq \bar{U} \text { is countable and } \\
C \subseteq \overline{c o}\left(\{0\} \cup R_{\mu}(C)\right) \text {, then } \bar{C} \text { is compact). }
\end{array}\right.
$$

Also if (2.3b) occurs we could replace (2.6) with (2.7) and

$$
\left\{\begin{array}{l}
\text { if } C \subseteq \bar{U} \text { is countable and } C \subseteq H(C \times[0,1]), \\
\text { then } \bar{C} \text { is compact. }
\end{array}\right.
$$

Remark 2.2. Notice it is easy to establish the analogue of Theorem 2.1 and Theorem 2.2 for multivalued maps which are closed (i.e. have closed graph). We leave the details to the reader.

Next we establish a generalization of Mönch's fixed point theorem.

Theorem 2.3. Let $E$ be a quasicomplete metrizable locally convex linear topological space and let $\Omega$ be an open, convex subset of $E$ with $x_{0} \in \Omega$. Suppose there is a continuous map $F: \bar{\Omega} \rightarrow \bar{\Omega}$ with the property:

$$
\left\{\begin{array}{l}
C \subseteq \bar{\Omega} \text { is countable and } C \subseteq \overline{c o}\left(\left\{x_{0}\right\} \cup F(C)\right) \\
\text { implies } C \text { is relatively compact. }
\end{array}\right.
$$

Then $F$ has a fixed point in $\bar{\Omega}$. 
Proof. Let

$$
D_{0}=\left\{x_{0}\right\} \text { and } D_{n}=c o\left(\left\{x_{0}\right\} \cup F\left(D_{n-1}\right)\right) \text { for } n=1,2, \cdots .
$$

We claim $D_{n}$ is relatively compact for $n=1,2, \cdots$. If $E$ is complete this is clear since the continuous image of a compact set is compact and the closed convex hull of a compact set is compact in a complete Hausdorff locally convex linear topological space [11, p. 67]. Next recall the following four properties: (i) if $X$ is any Hausdorff locally convex space, the convex hull of a totally bounded subset of $X$ is totally bounded [10 p. 72], (ii) a subset of a metric space is compact if and only if it is complete and totally bounded [3, p. 275], (iii) in metric spaces closures of totally bounded sets are totally bounded, and (iv) in a Hausdorff locally convex space $X$ if $K$ is a compact subset of $X$, then $K$ is bounded and so also is $c o(K)$ [10] p. 38]. Now it is clear that $D_{n}$ is relatively compact for $n=1,2, \cdots$ if $E$ is quasicomplete since closed, bounded subsets of quasicomplete spaces are complete [note in fact we also have $D_{n}$ is relatively compact even if $E$ is not metrizable].

Notice

$$
D_{0} \subseteq D_{1} \subseteq \cdots \subseteq D_{n-1} \subseteq D_{n} \subseteq \cdots \subseteq \bar{\Omega} .
$$

Also since compact metric spaces are separable, each $D_{n}$ is separable so there is a sequence of countable sets $\left\{C_{n}\right\}$ with $\overline{C_{n}}=\overline{D_{n}}$ for $n=0,1, \cdots$. Let

$$
D=\bigcup_{n=0}^{\infty} D_{n} \text { and } C=\bigcup_{n=0}^{\infty} C_{n}
$$

It is immediate since $\left(D_{n}\right)$ is increasing that

$$
D=\bigcup_{n=0}^{\infty} D_{n}=\bigcup_{n=1}^{\infty} \operatorname{co}\left(\left\{x_{0}\right\} \cup F\left(D_{n-1}\right)\right)=c o\left(\left\{x_{0}\right\} \cup F(D)\right) .
$$

Also since

$$
\bigcup_{n=0}^{\infty} D_{n} \subseteq \bigcup_{n=0}^{\infty} \overline{D_{n}} \subseteq \overline{\bigcup_{n=0}^{\infty} D_{n}}
$$

we have

$$
\overline{\bigcup_{n=0}^{\infty} \overline{D_{n}}}=\overline{\bigcup_{n=0}^{\infty} D_{n}}=\bar{D} \text { and } \overline{\bigcup_{n=0}^{\infty} \overline{D_{n}}}=\overline{\bigcup_{n=0}^{\infty} \overline{C_{n}}}=\overline{\bigcup_{n=0}^{\infty} C_{n}}=\bar{C} .
$$

The continuity of $F$ implies

$$
F(D) \cup\left\{x_{0}\right\} \subseteq F(\bar{D}) \cup\left\{x_{0}\right\} \subseteq \overline{F(D) \cup\left\{x_{0}\right\}} \subseteq \overline{c o}\left(F(D) \cup\left\{x_{0}\right\}\right)
$$

and so

$$
\overline{c o}\left(F(D) \cup\left\{x_{0}\right\}\right)=\overline{c o}\left(F(\bar{D}) \cup\left\{x_{0}\right\}\right) .
$$

Then (2.10), (2.11) and (2.12) imply

$$
\begin{aligned}
C \subseteq \bar{C}=\bar{D} & =\overline{c o}\left(F(D) \cup\left\{x_{0}\right\}\right)=\overline{c o}\left(F(\bar{D}) \cup\left\{x_{0}\right\}\right) \\
& =\overline{c o}\left(F(\bar{C}) \cup\left\{x_{0}\right\}\right)=\overline{c o}\left(F(C) \cup\left\{x_{0}\right\}\right) .
\end{aligned}
$$

Since $C$ is countable we have from (2.9) that $\bar{C}$ is compact. Hence $\bar{D}$ is compact. Also notice from (2.13) that

$$
\bar{D}=\overline{c o}\left(F(\bar{D}) \cup\left\{x_{0}\right\}\right)
$$


and so $F(\bar{D}) \subseteq \bar{D}$. We may apply the Schauder-Tychonoff Theorem to deduce that $F$ has a fixed point in $\bar{D}$.

Remark 2.3. Note that (2.9) could be replaced by the more general assumption:

$$
\left\{\begin{array}{l}
C \subseteq \bar{\Omega} \text { is countable and } \bar{C}=\overline{c o}\left(\left\{x_{0}\right\} \cup F(C)\right) \\
\text { implies } \bar{C} \text { is compact. }
\end{array}\right.
$$

Note also that it is enough in Theorem 2.3 to assume $E$ is a quasicomplete Hausdorff locally convex linear topological space where compact spaces are separable.

Remark 2.4. There is an analogue of Theorem 2.3 for multivalued u.s.c. maps $F$ which have the property that $F(\bar{A}) \subseteq \overline{F(A)}$ for any subset $A$ of $E$. We leave the details to the reader.

Our next result is the analogue of Theorem 2.3 for closed sets.

Theorem 2.4. Let $E$ be a quasicomplete metrizable locally convex linear topological space and let $Q$ be a closed, convex subset of $E$ with $x_{0} \in Q$. Suppose there is a continuous map $F: Q \rightarrow Q$ with the property:

$$
\left\{\begin{array}{l}
C \subseteq Q \text { is countable and } C \subseteq \overline{c o}\left(\left\{x_{0}\right\} \cup F(C)\right) \\
\text { implies } C \text { is relatively compact. }
\end{array}\right.
$$

Then $F$ has a fixed point in $Q$.

Proof. Essentially the same reasoning as in Theorem 2.3 establishes this. However, here we provide a second proof. By Dugundji's extension theorem [12] there is a continuous map $F^{\star}: E \rightarrow Q$ with $F^{\star}(x)=F(x)$ for $x \in Q$. Let $C \subseteq E$ be countable and

$$
C \subseteq \overline{c o}\left(\left\{x_{0}\right\} \cup F^{\star}(C)\right) .
$$

Since $F^{\star}(C) \subseteq Q$ we have from (2.15) that $C \subseteq Q$ and as a result $F^{\star}(C)=$ $F(C)$. We may now use (2.14) to deduce that $C$ is relatively compact. Next apply Theorem 2.3 to $F^{\star}$ with $\Omega=E$ to deduce the existence of an $x \in E$ with $x=F^{\star}(x)$. Since $F^{\star}(x) \in Q$ we must have $x \in Q$ and so $x=F^{\star}(x)=F(x)$.

Our next result is an example of the zero map being essential in $M_{\partial U}(\bar{U}, E)$.

Theorem 2.5. Let $E$ be a quasicomplete metrizable locally convex linear topological space and let $U$ be an open subset of $E$ with $0 \in U$. Then the zero map is essential in $M_{\partial U}(\bar{U}, E)$.

Proof. Let $\theta \in M_{\partial U}(\bar{U}, E)$ with $\left.\theta\right|_{\partial U}=0$. We must show that there exists $x \in U$ with $\theta(x)=x$. Let $Q=\overline{c o}(\theta(\bar{U}))$ and let $F: Q \rightarrow Q$ be given by

$$
F(x)=\left\{\begin{array}{l}
\theta(x), \quad x \in \bar{U}, \\
0, \quad \text { otherwise. }
\end{array}\right.
$$

Now $0 \in Q, F: Q \rightarrow Q$ is continuous and satisfies (2.14) (with $x_{0}=0$ ). To see this let $C \subseteq Q$ be countable with $C \subseteq \overline{c o}(\{0\} \cup F(C))$. Then

$$
C \subseteq \overline{c o}(\{0\} \cup \theta(\bar{U} \cap C)) .
$$

Notice $C \cap \bar{U} \subseteq Q$ is countable and

$$
C \cap \bar{U}(\subseteq C) \subseteq \overline{c o}(\{0\} \cup \theta(\bar{U} \cap C)) .
$$


Now since $\theta \in M_{\partial U}(\bar{U}, E)$ we have that $\overline{C \cap \bar{U}}$ is compact. Then since $\theta$ is continuous, $\theta(\overline{C \cap \bar{U}})$ is compact, and now since $E$ is a quasicomplete metrizable locally convex space, $\overline{c o}(\{0\} \cup \theta(\overline{C \cap \bar{U}}))$ is compact. Thus since

$$
C \subseteq \overline{c o}(\{0\} \cup \theta(\overline{C \cap \bar{U}}))
$$

we have that $\bar{C}$ is compact. Consequently $F: Q \rightarrow Q$ satisfies (2.14) (with $\left.x_{0}=0\right)$. Theorem 2.4 guarantees that there exists $x \in Q$ with $F(x)=x$. Now if $x \notin U$ we have $0=F(x)=x$, which is a contradiction since $0 \in U$. Thus $x \in U$ so $x=F(x)=\theta(x)$.

Combining Theorem 2.1 and Theorem 2.5 gives the following nonlinear alternative of Leray-Schauder type for Mönch type maps.

Theorem 2.6. Let $E$ be a quasicomplete metrizable locally convex linear topolog$i c a l$ space and let $U$ be an open subset of $E$ with $0 \in U$. Suppose $F \in M(\bar{U}, E)$ satisfies (2.2). Then $F$ is essential in $M_{\partial U}(\bar{U}, E)$ (in particular $F$ has a fixed point in $U)$.

\section{REFERENCES}

[1] R.P. Agarwal and D. O'Regan, Nonlinear essential maps of Mönch, 1-set contractive demicompact and monotone $(S)_{+}$type, Journal of Applied Mathematics and Stochastic Analysis, to appear.

[2] C. Corduneanu, Integral equations and applications, Cambridge Univ. Press, Cambridge, 1991. MR 92h:45001

[3] R. Engelking, General topology, Heldermann Verlag, Berlin, 1989. MR 91c:54001

[4] A. Granas, Sur la méthode de continuité de Poincare, C.R. Acad. Sci. Paris, 282(1976), 983-985. MR 53:11664

[5] D. Guo, V. Lakshmikantham and X. Liu, Nonlinear integral equations in abstract spaces, Kluwer Acad. Publishers, Dordrecht, 1996. MR 98a:45001

[6] G. Köthe, Topological vector spaces, Springer Verlag, New York, 1983.

[7] H. Mönch, Boundary value problems for nonlinear ordinary differential equations of second order in Banach spaces, Nonlinear Analysis, 4(1980), 985-999. MR 82c:34075

[8] D. O'Regan, Fixed points and random fixed points for weakly inward approximable maps, Proc. Amer. Math. Soc., 126(1998), 3045-3053. MR 99a:47084

[9] R. Precup, Generalized topological transversality and existence theorems, Libertas Mathematica, 11(1991), 65-79. MR 93a:54037

[10] W. Rudin, Functional analysis, McGraw Hill, New York, 1973. MR 51:1315

[11] F. Treves, Topological vector spaces, distributions and kernels, Academic Press, New York, 1967. MR 37:726

[12] E. Zeidler, Nonlinear functional analysis and its applications, I, Springer Verlag, New York, 1986. MR 87f: 47083

Department of Mathematics, National University of Singapore, 10 Kent Ridge CresCEnt, Singapore 119260

Department of Mathematics, National University of Ireland, Galway, Ireland

E-mail address: Donal.ORegan@nuigalway.ie 